\title{
External Locking Plate Fixation for a Femoral Shaft Fracture in an Unhealthy Patient with Deformity
}

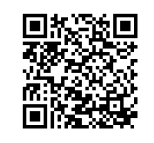

\author{
Paul Gagnet*, Daniel Paull and Nabil Ebraheim \\ Department of Orthopaedic Surgery, University of Toledo College of Medicine and Life Sciences, Spain
}

Submission: April 11, 2018; Published: April 24, 2018

*Corresponding author: Paul Gagnet, Department of Orthopaedic Surgery, University of Toledo College of Medicine and Life Sciences, 3000 Arlington Avenue, Toledo, Spain, Tel: 419-383-4000; Email: Paul.gagnet@rockets.utoledo.edu

\begin{abstract}
Multiple case studies have been performed at our institution, and other institutions on the subject of external locking plates (LCP) utilized in tibial fractures. Reports of utilizing an external LCP for fractures of the femur are very rare. This case presents a 22-year old male patient with a displaced distal mid-shaft femoral spiral fracture. Past medical history included failure to thrive, permanent non weight bearing status, and femoral bone deformity. The patient's femoral deformity and health status precluded him from intramedullary nailing and ORIF. The patient was treated with closed reduction and subsequent placement of an external locking compression plate. The external locking plate is less bulky than a standard external fixator and makes it advantageous for transfer of the patient, changing of clothes, and sleeping. We found that external locking plate fixation of a femoral shaft fracture is a viable alternative to standard external fixation in a patient that is unhealthy and has a femoral bone deformity present.
\end{abstract}

Keywords: External locking plate; External fixation; Femur

\section{Introduction}

Femoral shaft fractures are often the result of high-energy trauma but can also occur in low-energy trauma if significant bone pathology is present. The standard of care for femoral shaft fractures is placement of an intramedullary nail [1]. Placement of an intramedullary nail may not always be possible due to various contraindications including patient health status, coronal fractures, or deformity of the femur bone itself. When an intramedullary nail cannot be placed, and the patient has significant health problems, and the skin overlying the fracture site is compromised, a viable alternative is external fixation.

Kloen was the first to describe external locking compression plates (LCPs) as an alternative to standard external fixation [2]. Although other case studies have been performed at our institution, and other institutions on the subject of external locking plates utilized in tibial fractures [3-5], the reports are very rare on the subject of utilizing an external LCP for fractures of the femur. The concern with standard external fixation of the femur, is that the construct is not robust enough to allow adequate long term healing. This report presents a patient with a displaced distal mid shaft femoral spiral fracture. Due to the patient's frailty, failure to fully thrive, permanent non weight bearing status, and femoral bone deformity, the treatment consisted of closed reduction and subsequent placement of an external locking compression plate for fixation.

\section{Case Report}

A 22-year old male presented to the emergency department with acute onset of right thigh pain after his caretaker lifted him out of bed. Past medical history included chronic right hip dislocations, MRDD, growth delay, and seizure disorder due to anoxic brain injury. Examination revealed a shortened right lower extremity with tenting of the skin on the lateral aspect of the thigh with significant bruising, without open fracture. Sensation and motor function were unable to be obtained due to patient's non-verbal status and inability to follow commands. Radiographs revealed a displaced distal femoral shaft fracture in a spiral configuration. 5lbs of Bucks traction was placed on the lower right limb until the patient underwent surgery the following day.

Once in the operating room, his right femoral shaft fracture was reduced and held using traction. The plate used was an ITS 9 hole right distal femoral locking plate that was flipped around such that the distal end was now proximal. The plate was placed 2-3cm off the skin, and a plastic spacer of the same thickness was placed in between the skin and the plate. This was done in order to maintain the distance between the skin and the plate. Stab incisions through the skin were made, and eight $4.5 \mathrm{~mm}$ cortical locking screws were used, four proximal to and four distal to the fracture site respectively (Figure 1c). Care was taken all the 
while to maintain the reduction and distance from the plate to the skin. Post-operatively, the patient had multiple episodes of hypotension and tachycardia. The patient then stabilized over the next few days, and was discharged from the hospital on postoperative day four. The patient remained non-weight bearing, and received follow up imaging every 2-3 weeks for the next ten weeks. The radiographs showed good callus formation at 9 weeks post-operatively. At ten weeks after the initial surgery, the external locking plate and screws were removed under general anesthesia (Figure 1d). The patient was followed for two more months, healed his fracture fully, and continued to do well.

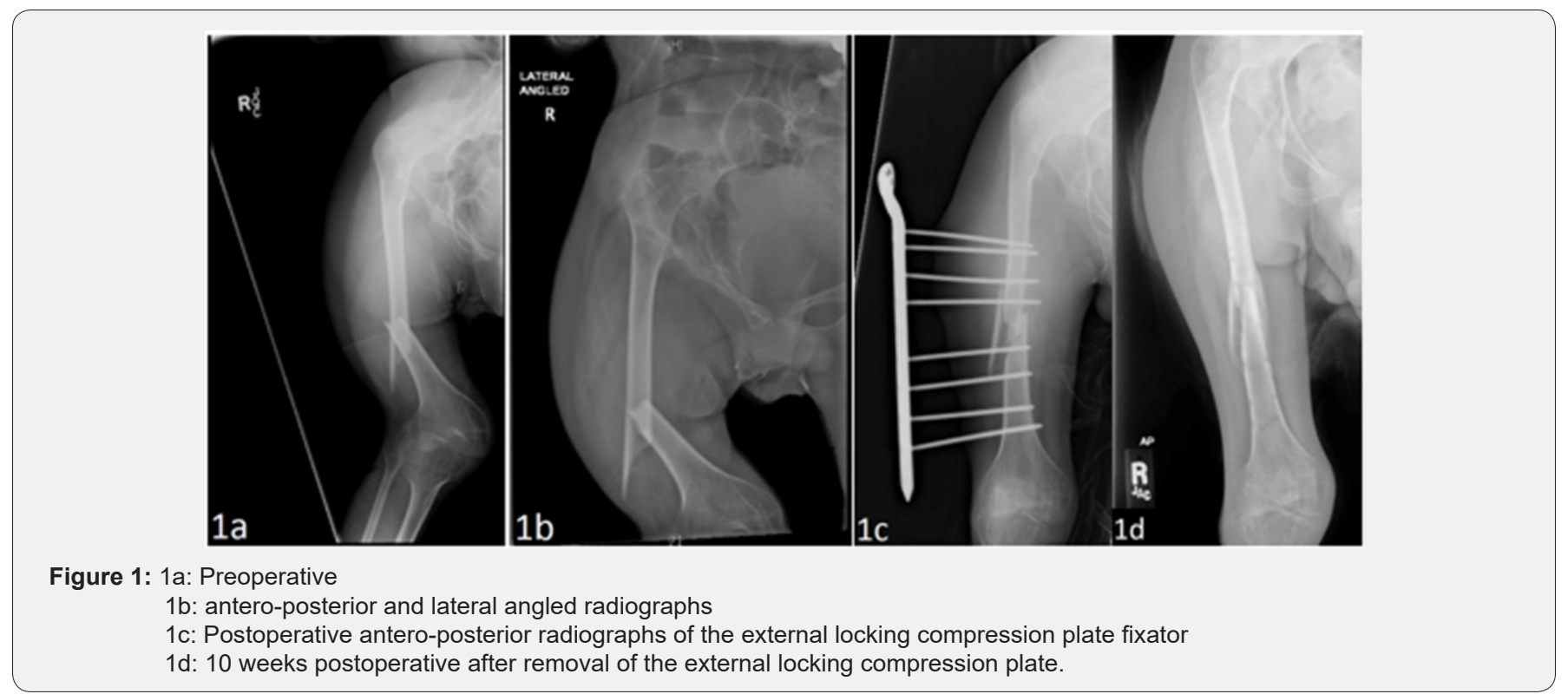

\section{Discussion}

A difficulty in treating femoral shaft fractures lies within a patient who is unhealthy, has poor skin quality overlying the fracture site, and has significant bone deformity. The deformity makes the passage of a standard intramedullary nails difficult, and precluded our patient from this surgical technique. The patient's health and skin status placed him at significant risk for infection with open reduction and internal plating. While not commonly used, external plate fixation can be a viable option in fixation of femoral shaft fractures. The rigid plate and multiple locking screws provide a stable construct, which can be a good alternative to standard external fixation. One of the advantages of external plating is that it is a much less bulky frame compared to that of standard external fixators. This makes the process of changing clothes, sleeping, and general care much easier. It also eliminates the sharp points present on standard external fixators which can eliminate possible harm to adjacent body structures during movement [6]. The previous factors are even more of a concern when the patient has limited mental awareness and requires total care, as in the case of our patient.

\section{Conclusion}

To conclude, external plate fixation of a femoral shaft fracture is a viable alternative in a patient who is unhealthy, had bone deformity, skin compromise, and who might not tolerate a standard external fixator.

\section{Conflict of Interest}

No conflict of interest.

\section{References}

1. Bhandari M, Guyatt G, Khera V, Kulkamai A, Sprague S, et al. (2003) Operative management of lower extremity fractures in patients with head injuries. Clin Orthop Relat Res (407): 187-198.

2. Kloen P (2009) Supercutaneous plating: use of a locking compression plate as an external fixator. J Orthop Trauma 23(1): 72-75.

3. Ebraheim N, Carroll T, Hanna M, Zhang J, Liu J (2014) Staged Treatment of Proximal Tibial Fracture Using External Locking Compression Plate. Orthop Surg 6(2): 154-157.

4. Ma CH, Tu YK, Yeh JH, Yang SC, Wu CH (2011) Using external and internal locking plates in a two-stage protocol for treatment of segmental tibial fractures. J Trauma 71(3): 614-619.

5. Apivatthakakul T, Sananpanich K (2007) The locking compression plate as an external fixator for bone transport in the treatment of a large distal tibial defect: a case report. Inj 38: 1318-1325.

6. CY Woon, MK Wong, TS Howe (2010) LCP external fixation - external application of an internal fixator: two cases and a review of the literature. J Ortho Surg Res 5: 19. 


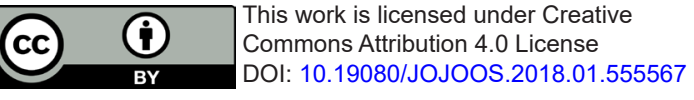

Your next submission with Juniper Publishers will reach you the below assets

- Quality Editorial service

- Swift Peer Review

- Reprints availability

- E-prints Service

- Manuscript Podcast for convenient understanding

- Global attainment for your research

- Manuscript accessibility in different formats

( Pdf, E-pub, Full Text, Audio)

- Unceasing customer service

Track the below URL for one-step submission https://juniperpublishers.com/online-submission.php 